\title{
Properties of the Cholesteric Liquid Crystals of Polyribonucleotide Complexes
}

\author{
Eisaku IIzUKA \\ Institute of High Polymer Research, Faculty of Textile Science \\ and Technology, Shinshu University, Ueda 386, Japan.
}

(Received October 6, 1977)

\begin{abstract}
Properties of the cholesteric solutions of $\operatorname{poly}(A) \cdot \operatorname{poly}(U)$ and $\operatorname{poly}(A)$. 2 poly $(U)$ were investigated by means of circular dichroism and diffraction of laser light. These liquid crystals show a negative form-CD as well as a positive induced-CD of acridine orange incorporated into solution, suggesting that the helical sense of the cholesteric structures is right-handed. In a static magnetic field the pitch of the cholesteric structures changes with the field strength and this change varies with the angle that the helical axis of the cholesteric texture makes with respect to the direction of magnetic field. The magnetic susceptibility of these polyribonucleotide complexes appears to be higher in a direction parallel to the polymer helices than in a direction perpendicular to the polymer helices.

KEY WORDS Polyribonucleotide Complex / Cholesteric Liquid Crystal / Circular Dichroism / Helical Sense / Magnetic Susceptibility /
\end{abstract}

In a preceding paper $^{1}$ it was reported that double-stranded helices of $\operatorname{poly}(\mathbf{A}) \cdot \operatorname{poly}(\mathbf{U})$, $\operatorname{poly}(G) \cdot \operatorname{poly}(C)$ and $\operatorname{poly}(C) \cdot \operatorname{poly}(\mathrm{I})$ and triplestranded helices of poly(A).2poly(U) and poly (A) 2 poly $(I)$ in concentrated solution form nematic liquid crystals in which $\operatorname{poly}(\mathrm{C}) \cdot \operatorname{poly}(\mathrm{I})$ includes some spherulitic structures. The molecular assemblies of these polyribonucleotide complexes are rod-like and in these molecular assemblies (molecular clusters) the polymer helices are aligned parallel to the rod axes. The rod-like molecular clusters align themselves with their long axes parallel to the direction of shearing stresses and show negative polarization dichroism as well as strong, negative birefringence especially in $\operatorname{poly}(\mathrm{A}) \cdot \operatorname{poly}(\mathrm{U})$ and $\operatorname{poly}(\mathrm{A})$. 2 poly $(U)$. On standing for several weeks the liquid crystals of these two polymer complexes are converted from the nematic to the cholesteric type, which reverses to the nematic type under shearing stresses. The helical pitch of the cholesteric structure is longer in $\operatorname{poly}(\mathrm{A}) \cdot 2 \mathrm{poly}(\mathrm{U})$ than in $\operatorname{poly}(\mathrm{A}) \cdot \operatorname{poly}(\mathrm{U})$ at a fixed polymer con- centration and is proportional to $c_{\mathrm{v}}^{-1.1}$ where $c_{\nabla}$ is the volume fraction of the polymers. This result contrasts to that of Robinson's on poly( $\gamma$-benzyl L-glutamate) where the helical pitch is proportional to $c_{\mathrm{v}}{ }^{-2}$ and the rod-like polymer molecules are considered to be packed in a way not completely hexagonal nor completely random. ${ }^{2}$ The pitch of the cholesteric structures of these polyribonucleotide complexes first shows a gradual increase with the temperature comparable to the coefficient of the linear expansion of the polymer solution. This is followed by a sharp increase in the expansion of the pitch and eventually the liquid crystals melt to become an isotropic solution; the melting point of the liquid crystals is higher in a more concentrated solution. It has also been shown that these cholesteric structures are susceptible to magnetic influence.

The purpose of this paper is to resport on the helical sense of these cholesteric liquid crystals and also on some new findings about the magnetic-field effect on these cholesteric textures. 


\section{E. IIzuKA}

\section{EXPERIMENTAL}

\section{Materials}

Potassium salt of polyadenylate (poly(A), control \#65) and ammonium salt of polyuridylate (poly(U), control \#11-65A-308), each distributed by the Miles Laboratories, Inc., were donated by Professor J. T. Yang and used without further purification. They were dissolved in $0.08 \mathrm{M}$ $\mathrm{NaCl}$ and $0.02 M$ sodium citrate plus citric acid with a $\mathrm{pH}$ of $7.0^{3}$ and mixed up to obtain two kinds of polymer complexes, poly $(A) \cdot \operatorname{poly}(U)$ and $\operatorname{poly}(\mathrm{A}) \cdot 2 \operatorname{poly}(\mathrm{U})$. These polymer solutions had polymer concentrations of $5.5 \mathrm{vol} \%$ or more and were kept for a few weeks to insure full growth of the nematic liquid crystals. They were then introduced into quartz cells of $1-\mathrm{mm}$ pathlength and stored for another few weaks before measurements. By that time the solutions had showed brilliant, iridescent colors which originated from the selective reflection of light due to the cholesteric textures. Visible equidistant parallel lines, associated with an oscilating value of retardation, ${ }^{4}$ appeared as well under crossed polarizers in some cases (Figure 1). A quartz spacer was inserted into each optical cell to shorten the lightpath to about $0.1 \mathrm{~mm}$ for the circular dichroism (CD) measurements.

By proper choice of salt concentration and mole fraction of polyribonucleotides, as in the case of this study, $\operatorname{poly}(\mathrm{A}) \cdot \operatorname{poly}(\mathrm{U})$ and $\operatorname{poly}(\mathrm{A})$. 2 poly $(\mathrm{U})$ can be prepared as double- and triplestranded helices, respectively in dilute solution. ${ }^{5}$ The preparations of double- and triple-stranded complexes have been described elsewhere also by Sarker and Yang. ${ }^{6,7}$ At neutral $\mathrm{pHs}$, poly(A)

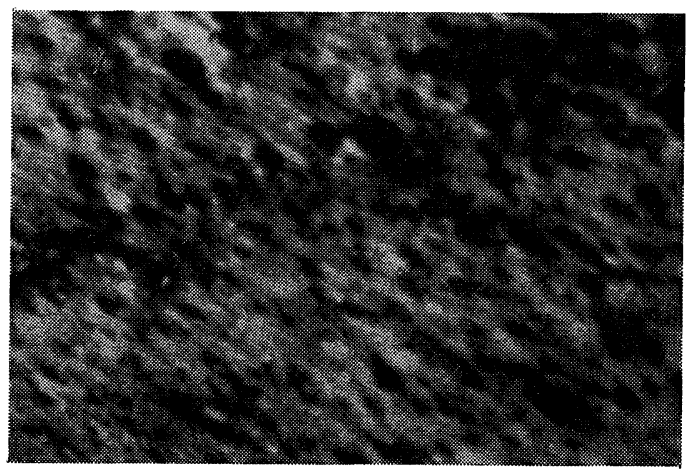

Figure 1. Polarizing micrograph of a 5.5-\% cholesteric solution of $\operatorname{poly}(\mathbf{A}) \cdot 2 \operatorname{poly}(\mathbf{U})$. and poly(U) do not form liquid crystals in concentrated solution. ${ }^{1}$ The cholesteric pitch is longer in $\operatorname{poly}(\mathbf{A}) \cdot 2 \operatorname{poly}(\mathbf{U})$ than in $\operatorname{poly}(\mathbf{A})$. poly $(\mathrm{U}){ }^{1}$ Thus the polyribonucleotide strands in dilute solution also hold in concentrated (liquid crystalline) solution.

\section{Methods}

The CD of the concentrated solutions was measured with a Jasco ORD/UV-5 spectropolarimeter with the $\mathrm{CD}$ attachment. The measurements were carried out on solutions with and without acridine orange (AO) added to solution by 1 part against about 100 parts of the polymers. The AO was of reagent grade and used without further purification. The incident light through cholesteric texture, which can be looked upon as being composed of a number of equally spaced planes (molecular layers), is diffracted in accordance with the Bragg equation with which the helical pitch is calculated. ${ }^{8}$ The cholesteric solutions were placed, with the surfaces of the optical cell parallel to the direction of magnetic field, between the poles of an electromagnet, a modified model of JM-151 manufactured by Japan Electron Optics Laboratory Co., Ltd. having the maximum output of 25 kilogausses and light diffraction patterns were photographed with a 2-mW He-Ne gas laser $(6328 \mathrm{~A})$ as the light source. All the experiments were done at room temperature unless stated otherwise.

\section{RESULTS AND DISCUSSION}

\section{Helical Sense of the Cholesteric Structures}

Robinson suggested that the cholesteric color of concentrated polypeptide solutions is originated from the selective reflection of circularly polarized light of one sense determined by the helical sense of the cholesteric structure ${ }^{8}$ and showed, using a quarter plate, that the light reflected by a concentrated ethyl acetate solution of poly( $\gamma$-ethyl L-glutamate) (PELG) was right circularly polarized. ${ }^{9}$ Along with this suggestion, Tachibana and Oda detected a broad, positive CD centered around $550 \mathrm{~nm}$ in solid films of poly $(\gamma$-methyl D-glutamate) (PMDG) cast and dried from a chloroform solution and showed that these films reflected selectively left circularly polarized light, concluding that the helical sense of the cholesteric structure was left-handed. ${ }^{10}$ 
They noticed that solid films of the L-isomer of this polypeptide (PMLG) gave the CD curves of the opposite sense. Tsuchihashi and his coworkers found that the sign of $C D$ was positive in a vinyl acetate solution of PELG. ${ }^{11}$ This indicated that the selectively reflected light of PELG was left-circularly polarized in contrast with Robinson's result and they concluded that the helical sense of the lyotropic cholesteric texture in the PELG solution depended on the solvent in which the polyglutamate was dissolved. This $C D$, due to the selective reflection by the cholesteric texture (form-CD), has become the means for Tsuchihashi and his coworkers ${ }^{11}$ to determine the helical sense of lyotropic cholesteric structures on the basis of the observed CD sign in comparison with the results of Saeva and Wysocki ${ }^{12-14}$ on thermotropic cholesteric structures.

The CD spectra of the cholesteric liquid crystals of $\operatorname{poly}(A) \cdot \operatorname{poly}(U)$ having a very high polymer concentration ( $\mathrm{ca} .30 \mathrm{vol} \%)$ are shown in Figure 2, where a broad, negative band centered around $670 \mathrm{~nm}$ is observed near room temperature. This band is due to the selective reflection of circularly polarized light of one sense because the poly(A).poly(U) solution has no intrinsic absorption band near $670 \mathrm{~nm}$. Furthermore, the center of the band appears to make the red shift with the temperature in accordance with the increase of pitch, $P$, of the cholesteric structure. This is because the wavelength of the maximum reflectivity, $\lambda_{\mathrm{m}}$, is related to the pitch in the form, ${ }^{15} \lambda_{\mathrm{m}}=n \times P$, where $n$ is

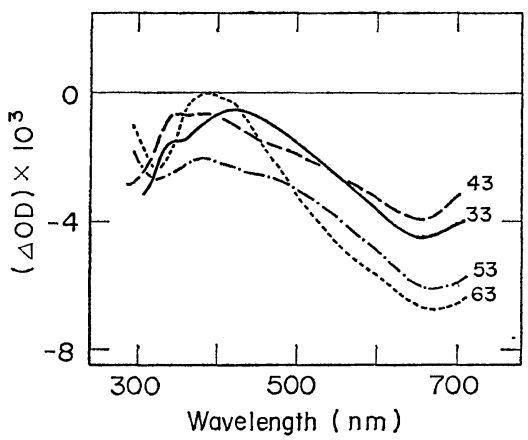

Figure 2. Form-CD spectra of the cholesteric solution of poly(A) p poly(U). Polymer concentration, ca. 30 vol\%. The numbers on the curves refer to the temperature in ${ }^{\circ} \mathrm{C}$. the average refractive index of the medium. The pitch of this cholesteric solution is estimated to be about $500 \mathrm{~nm}$, or a little bit less, from the relationship ${ }^{1}$ between the pitch and the polymer concentration. (Such a small pitch could not be determined directly under the microscopic stage.) The value of $n$ was 1.4 , which is quite reasonable. The intensity of the $\mathrm{CD}$ becomes stronger with the temperature, but it is considered to start decreasing when the cholesteric texture begins to be destroyed at a still higher temperature. One plausible explanation for this temporary increase in the $\mathrm{CD}$ intensity is that the possible decrease in viscosity enhances the formation of cholesteric structure. The negative sign of the form-CD observed indicates that the selectively reflected light is right circularly polarized and suggests that the helical sense of the cholesteric structure of $\operatorname{poly}(A) \cdot \operatorname{poly}(U)$ is righthanded (see ref 10). As for poly (A).2poly(U), a solution having the helical pitch that makes the form-CD observable with the spectropolarimeter used in this study could not be prepared.

Saeva and Wysocki found that archiral dye molecules become optically active when incorporated into thermotropic liquid crystals of the cholesteric type accompanying a strong CD induced in the wavelength region of the absorption bands of the dye molecules. ${ }^{12,13}$ A general rule for the sign relationship between the form-CD and the induced-CD was proposed for solutes in cholesteric mesophases composed of cholesteryl derivatives that may change as the components of the cholesteric mesophase are varied. ${ }^{13}$ Tsuchihashi and his coworkers posturated that the induced-CD was brought about by the dissymmetric field of the cholesteric helix. ${ }^{16}$ They observed that the sign of the induced-CD of AO molecules was negative when incorporated into a dichloromethane solution of poly $(\gamma$-benzyl-Dglutamate) (PBDG) and positive when incorporated into a tetrachloroethane solution and suggested that the helical sense of the cholesteric structure was dependent on the solvent used as was shown for PELG by the sign of the formCD.

The CD spectra of the cholesteric liquid crystals of $\operatorname{poly}(\mathrm{A}) \cdot \operatorname{poly}(\mathrm{U})$ and $\operatorname{poly}(\mathrm{A}) \cdot 2 \operatorname{poly}(\mathrm{U})$ in which AO molecules are incorporated are shown in Figure 3, where a positive band having a 


\section{E. IrzuKa}

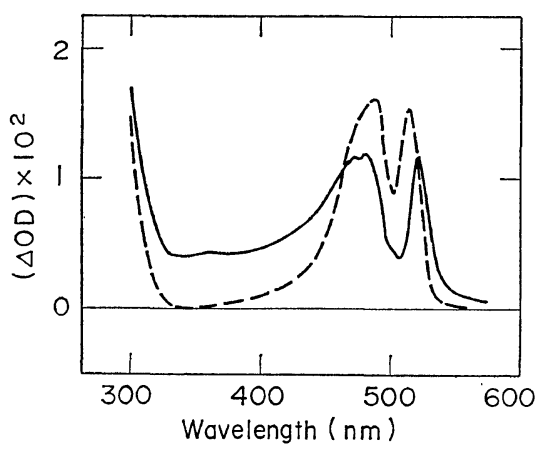

Figure 3. Induced-CD spectra of acridine orange incorporated into the cholesteric solutions of polyribonucleotide complexes. Full curve, poly(A). $\operatorname{poly}(U)$ and broken curve, $\operatorname{poly}(A) \cdot 2 \operatorname{poly}(\mathrm{U})$. Polymer concentration, 10.5 vol\%.

dip around $500 \mathrm{~nm}$ is found in both polymer complexes. The $C D$ was also detected around this wavelength in isotropic solutions of these polymer complexes; however, its intensity was much weaker (about $1 / 100$ ) than when in cholesteric solution. No $C D$ band due to the selective reflection existed near this wavelength. Therefore, the observed CD is undoubtedly induced in AO molecules incorporated into the cholesteric texture. The sign of this induced-CD is opposite to that of the form-CD and coincides with Saeva's observation. ${ }^{12}$ The fact that the sign of the induced-CD is the same for both polymer complexes may indicate that their cholesteric helical sense are same.

Magnetic-Field Effects on the Cholesteric Structures

Meyer was able to derive equations for the cholesteric liquid crystal which are easily tested on the basis of Frank's theory of curvatureelasticity ${ }^{17}$ in molecularly uniaxial liquid crystals. ${ }^{18}$ In doing this he assumed that the layers of molecular alignment are either parallel or perpendicular to the electric or magnetic field. These equations give the pitch as a function of field strength, $F$, in which the electric or magnetic susceptibility, having two components, $\chi_{\mathrm{p}}$ and $\chi_{\mathrm{t}}$, parallel and perpendicular to the unit vector (which is defined to be parallel to the axis of molecular alignment at any point), respectively and moduli of torsional and bending strains, respectively $k_{22}$ and $k_{33}$, are also related.

In a static magnetic field the diffraction pattern of the cholesteric solutions undergoes time-dependent change which is also dependent on the angle that the direction of magnetic field makes with respect to the layers of molecular alignment as may be seen in Figure 4. This suggests that the cholesteric pith changes in the magnetic field, thus indicating the presence of an anisotropy of the magnetic susceptibility in the polyribonucleotide helices that causes the magnetic field to exert torques within the cholesteric structure. These magnetic-field induced torques compete with the elastic torques determining the internal structure of the cholesteric phase and consequently the cholesteric pitch changes to satisfy the condition of the minimum, total free energy of the system. ${ }^{18}$

Examples of the pitch change as a function of the field strength are shown in Figure 5. When the magnetic field is parallel to the layers of molecular alignment, the intensity of diffracted light from poly(A).2poly(U) liquid crystal decreases gradually with the field strength (the middle row in the figure) and this is the case also in $\operatorname{poly}(\mathbf{A}) \cdot \operatorname{poly}(\mathbf{U})$ liquid crystal (the upper row in Figure 4). Perturbations of the helical structure are thus known to be induced by the magnetic field; even the complete breakdown of the cholesteric structure in large field, 23 kilogausses and over, was observed in dilute cholesteric solutions such as a $8.1-\%$ poly(A). $\operatorname{poly}(\mathbf{U})$.

According to Meyer the cholesteric pitch, $P$, in the electric or magnetic field parallel to the molecular layers is given, as the first approximation, in the form: ${ }^{18}$

$$
P=P_{0}\left[1+\frac{\left(\chi_{\mathrm{p}}-\chi_{\mathrm{t}}\right)^{2} F^{4}\left(P_{0}\right)^{4}}{32(2 \pi)^{4} k_{22}^{2}}\right]
$$

where $P_{0}$ is the cholesteric pitch to be observed without external field. The change of pitch, $P-P_{0}$, is positive and proportional to $F^{4}$. The cholesteric pitch of the polyribonucleotide complexes in a magnetic field was calculated with the Bragg equation, ${ }^{8} m \lambda=2 d \sin \theta$, where $d$ is the half pitch $(P / 2), \theta$ is the angle between the planes (molecular layers) and the direction of the incident light, $m$ is the order of diffraction and $\lambda$ is the wavelength of light in the medium, that is, the wavelength in vacuum divided by the mean refractive index of the solution (1.4). The 

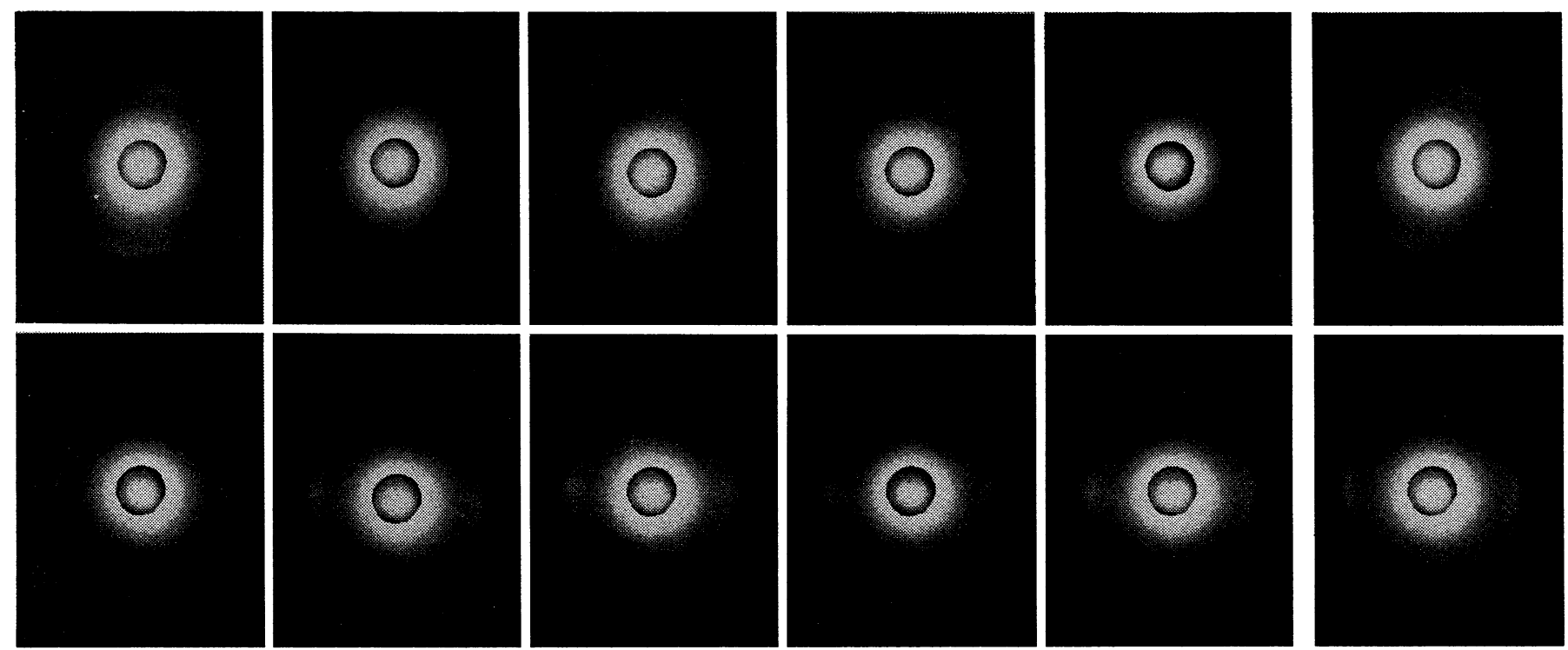

Figure 4. Time-dependence of the light diffraction patterns of the cholesteric solution of $\operatorname{poly}(A) \cdot \operatorname{poly}(U)$ in a magnetic field. Polymer concentration, $10.5 \mathrm{vol} \%$. Direction of the magnetic field (25 kilogausses), horizontal, that is, parallel (upper) and nearly perpendicular (lower) to the layers of molecular alignment. From left to right: $0,30,80,240,600 \mathrm{sec}$ after application of the field, and $180 \mathrm{sec}$ after removal of the field. 


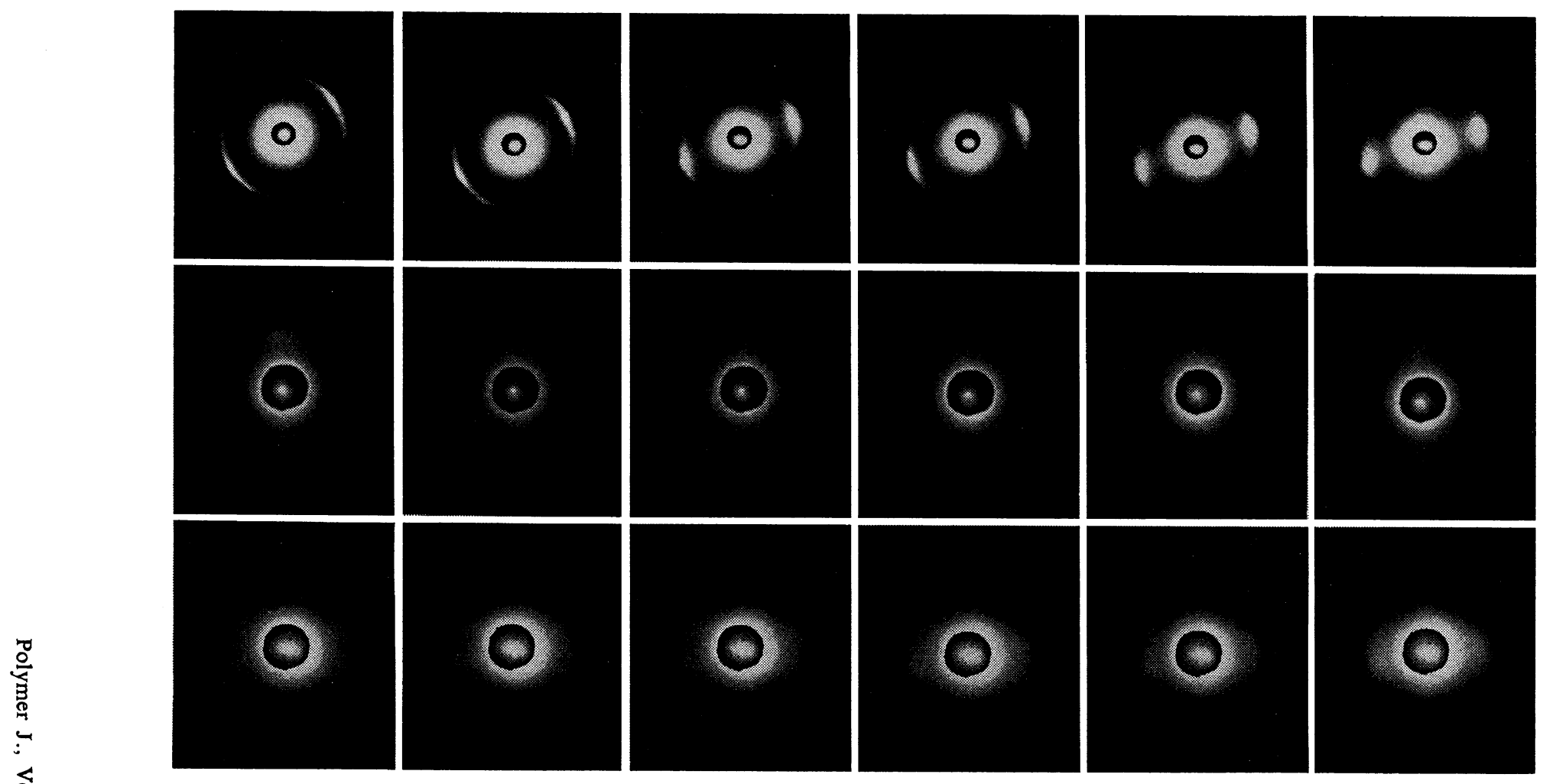

Figure 5. Dependence of the steady-state light diffraction patterns of the cholesteric solutions of polyribonucleotide complexes upon magnetic-field strength. Upper, 8.1-\% poly(A). poly(U); middle and lower, 14.9-\% poly(A).2poly(U). Direction of the magnetic field, horizontal (see the caption to Figure 4). Field strength: from left to right, 0, 10, 16, 20, 23 and 25 kilogausses. 


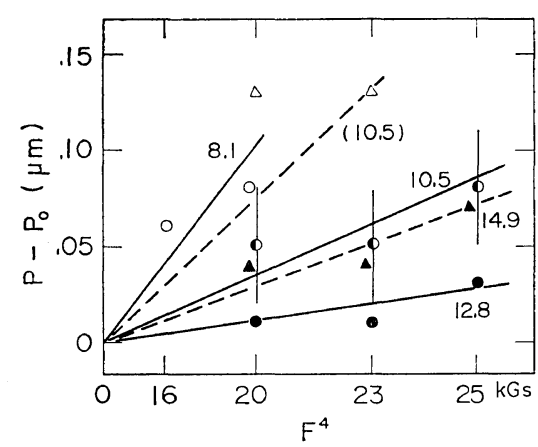

Figure 6. Change of the cholesteric pitch $v s$. field strength relationship when the direction of magnetic field is parallel to the layers of molecular alignment. Full lines, poly $(\mathrm{A}) \cdot \operatorname{poly}(\mathrm{U})$ and broken lines, $\operatorname{poly}(\mathbf{A}) \cdot 2 \operatorname{poly}(\mathbf{U})$. The numbers on the lines refer to the polymer concentration in vol $\%$.

change of pitch in the magnetic fields was then evaluated and plotted against $F^{4}$ as shown in Figure 6. Although no good linear relationship as observed in the cholesteric liquid crystals of polypeptides $^{19}$ was obtained, the values of $k_{22} /$ $\left(\chi_{\mathrm{p}}-\chi_{\mathrm{t}}\right)$ were only tentatively estimated and are shown in Table I. The difference in the values between $\operatorname{poly}(\mathrm{A}) \cdot \operatorname{poly}(\mathrm{U})$ and $\operatorname{poly}(\mathrm{A}) \cdot 2 \operatorname{poly}(\mathrm{U})$ may not suggest a real difference if any and the dependence of polymer concentration on this value is not clear either.

When the magnetic field is nearly perpendicular to the layers of molecular alignment, application of the magnetic field increases the intensity of diffracted light in both $\operatorname{poly}(\mathrm{A}) \cdot \operatorname{poly}(\mathrm{U})$ and $\operatorname{poly}(\mathrm{A}) \cdot 2 \operatorname{poly}(\mathrm{U})$ (the lower row in Figure 4 and the upper and the lower rows in Figure 5) as was observed by Muller ${ }^{20}$ in some cases. This observation can be explained by Meyer's theory assuming that $\chi_{\mathrm{t}}>\chi_{\mathrm{p}}$ as Meyer did in explaining Muller's observation. (For diamagnetic

Table I. Tentative values of $\left|k_{22} /\left(\chi_{\mathrm{p}}-\chi_{\mathrm{t}}\right)\right|$

\begin{tabular}{ccc}
\hline Preparation & $\begin{array}{c}\text { Polymer concn, } \\
\text { vol } \%\end{array}$ & $\left|k_{22} /\left(\chi_{\mathrm{p}}-\chi_{\mathrm{t}}\right)\right|$ \\
\hline & 8.1 & 1.6 \\
Poly(A)·poly(U) & 10.5 & 1.3 \\
& 12.8 & 1.4 \\
\hline Poly(A)·2poly(U) & 10.5 & $6.0^{\mathrm{a}}$ \\
& 14.9 & 3.4 \\
\hline
\end{tabular}

2 This value is only temporary.

Polymer J., Vol. 10, No. 3, 1978 substances as in this case, $\left|\chi_{\mathrm{p}}\right|>\left|\chi_{\mathrm{t}}\right| \cdot$.) Since the planer texture already satisfies approximately the condition of the lowest energy in the system, the magnetic field merely eliminates existing perturbations in the helical texture, making it more perfect and thus intensifying the diffracted light. It may also be noticed that the diffraction spots (or arcs) become broader in a higher magnetic field. In the magnetic field of 25 kilogausses this broadening corresponds to the scattering of pitch between 3.2 and $4.2 \mu \mathrm{m}$ (mean value 3.7 $\mu \mathrm{m})$ for a $8.1-\%$ poly $(\mathrm{A}) \cdot \operatorname{poly}(\mathrm{U})$. This suggests that there is a considerable range of pitch allowed to be present in a solution under magnetic field. The small broadening of the arcs without an external magnetic field corresponding to the pitch centering around $3.4 \mu \mathrm{m}$ is considered to be due mainly to the expanse of the incident laser beam (about $1 \mathrm{~mm}$ in diameter) and the scattering is apparent. As a result of eliminating perturbations, the cholesteric structure is now allowed to exist in regions where fluctuations of polymer concentration are present, causing the scattering of pitch. The mean pitch is larger in the magnetic field than in the case of no external magnetic field. This may appear incompatible with the idea that the helical texture is stabilized in magnetic field; however, the lower limit of the pitch is surely smaller than the initial pitch $(3.4 \mu \mathrm{m})$. One plausible explanation for this discrepancy may be that helical textures are newly formed mainly in regions where the polymer concentrations are lower than in regions having the mean polymer concentration.

Acknowledgments. I thank Professor J. T. Yang of Cardiovascular Research Institute, University of California, San Francisco for the donation of the polyribonucleotides used and many helpful suggestions.

\section{REFERENCES}

1. E. Iizuka and J. T. Yang, in "Liquid Crystals and Ordered Fluids," Vol. 3, J. F. Johnson and R.S. Porter, Eds., Plenum Publishing Corp., New York, N.Y., 1978, p 197.

2. C. Robinson, J.C. Ward and R. B. Beevers, Disc. Faraday Soc., 25, 29 (1958).

3. G. C. Chen and J. T. Yang, Biophys. Chem., 1, 62 (1973). 
4. C. Robinson, Trans. Faraday Soc., 52, 571 (1956).

5. G. Felsenfeld, D. R. Davies and A. Rich, J. Am. Chem. Soc., 79, 2023 (1957).

6. P. K. Sarker and J. T. Yang, J. Biol. Chem., 240, 2088 (1965).

7. P. K. Sarker and J. T. Yang, Biochemistry, 4, 1238 (1965).

8. C. Robinson, Tetrahedron, 13, 219 (1961).

9. C. Robinson, Mol. Cryst., 1, 467 (1966).

10. T. Tachibana and E. Oda, Bull. Chem. Soc. Jpn., 46, 2583 (1973).

11. N. Tsuchihashi, H. Nomori, M. Hatano and
S. Mori, ibid., 48, 29 (1975).

12. F. D. Saeva and J. J. Wysocki, J. Am. Chem. Soc., 93, 5928 (1971).

13. F. D. Saeva, ibid., 94, 5135 (1972).

14. F. D. Saeva, Mol. Cryst. Liq. Cryst., 18, 379 (1972).

15. de Vries, HL., Acta Cryst., 4, 219 (1951).

16. N. Tsuchihashi, H. Nomori, M. Hatano and S. Mori, Chem. Lett., 823 (1974).

17. F. C. Frank, Disc. Faraday Soc., 25, 19 (1958).

18. R. B. Meyer, Appl. Phys. Letters, 12, 281 (1968).

19. E. Iizuka, Polym. J., 4, 401 (1973).

20. J. H. Muller, Mol. Cryst., 2, 167 (1966). 